\title{
Effect of gel-forming gums on the intestinal unstirred layer and sugar transport in vitro
}

\author{
I T JOHNSON AND JENNIFER M GEE
}

\author{
ARC Food Research Institute, Colney Lane, Norwich
}

SUMMARY The effect of two gel-forming polysaccharide gums, guar gum and Na-carboxymethylcellulose (CMC), on glucose transport in vitro was investigated using everted sacs of rat jejunum. The gums were added to the mucosal bathing media to give apparent viscosities in the range 1-110 Pascal seconds $\times 10^{-3}, \mathrm{mPa} \cdot \mathrm{s}(\mathrm{cP})$. Serosal glucose transport fell steeply by about $60 \%$ as the viscosities of the mucosal media rose to $20 \mathrm{mPa} \cdot \mathrm{s}$, and levelled off thereafter. A similar effect was observed in sacs preincubated with guar gum (15 minutes) and exposed to glucose in a subsequent guar-free incubation. Glucose transport with and without the addition of guar gum was found to be sensitive to mucosal stirring, so that, when shaken at 130 oscillations per minute, sacs exposed to guar gum $(0.25 \%$, viscosity c.a. $16 \mathrm{mPa} \cdot \mathrm{s}(\mathrm{cP})$ transported glucose at a similar rate to sacs incubated without guar at 80 oscillations per minute. By measuring the time course for the establishment of osmotic induced potentials, it was shown that incubation with guar or CMC led to an increase in the apparent thickness of the unstirred fluid layer overlying the mucosa (guar-free thickness $=317 \pm 15 \mu$, guar treated thickness $=468 \pm 25 \mu$ ). It is suggested that the presence of a polysaccharide gum in the fluid film surrounding the villi increases its viscosity, and thus gives rise to a thickening of the rate-limiting unstirred layer. If such an effect occurs in vivo, this could contribute to the diminished post-prandial glycaemia observed in human subjects fed guar gum.

In recent years, a promising advance in the dietary management of diabetes has come about with the observation that post-prandial glycaemia in man is strongly influenced by dietary fibre. ${ }^{1}$ Studies with human subjects have demonstrated that glucose absorption is slowed by the simultaneous ingestion of viscous dietary gums such as guar gum, pectin, or methylcellulose, and this effect has been shown to increase with the viscosity of the ingested gum. ${ }^{23}$ Conversely, the reduction in hyperglycaemia associated with the ingestion of guar gum, the most effective material, is nullified by mild hydrolysis, which renders it non-viscous. ${ }^{3}$

The mechanism underlying the effect of viscous forms of dietary fibre is still a matter of controversy. Recently, Holt et al. ${ }^{4}$ have demonstrated that relatively large doses of guar gum and pectin markedly reduce the rate of gastric emptying in man, but their conclusion that this in itself is sufficient to account for the observed reduction of glucose absorption has been disputed. ${ }^{56}$ Earlier workers have suggested that the presence of viscous fibre in

Received for publication 25 November 1980 the small intestine may slow absorption at the mucosal surface, but little evidence exists for or against this proposition..$^{72}$ In the present study we have investigated the effect of viscous gums on glucose transport in vitro, thus avoiding the influence of intestinal motility and other systemic effects. We demonstrate that low levels of guar gum and sodium carboxymethylcellulose cause a pronounced reduction in glucose transport, and we show that this is probably brought about by an increase in the effectiveness of the mucosal diffusion barrier.

\section{Methods}

PHYSIOLOGICAL PREPARATIONS

Male Wistar strain rats $(150-250 \mathrm{~g})$ were allowed food and water ad libitum, before being killed by stunning and decapitation. Groups of everted jejunal sacs were prepared from each animal, filled with glucose-free Krebs bicarbonate buffer, and assigned randomly or by means of a latin square design to control and treatment incubation flasks. Glucose transport experiments were carried out in $25 \mathrm{ml}$ conical flasks containing $10 \mathrm{ml}$ of medium, 
incubated at $37^{\circ} \mathrm{C}$ in a shaking water bath at 80 oscillations per minute, unless otherwise stated. In some experiments a second incubation stage was introduced. Sacs preincubated in glucose free media were suspended in a jacketed organ bath, containing medium, gently stirred by a rising column of bubbles. For transmural potential difference measurements, sacs were ligatured over glass cannulae (ca. $2.5 \mathrm{~cm}$ in length) and suspended in $15 \mathrm{ml}$ glass centrifuge tubes containing incubation media, which were gassed continuously with $95 \% \mathrm{O}_{2}: 5 \% \mathrm{CO}_{2}$, and maintained at $37^{\circ} \mathrm{C}$ in a water bath.

\section{INCUBATION MEDIA}

All incubation media were prepared from Krebs bicarbonate buffer, pregassed with $95 \% \mathrm{O}_{2}: 5 \% \mathrm{CO}_{2}$, having a final $\mathbf{p H}$ of 7.4. Dispersions of guar gum (Sigma Ltd) and high viscosity sodium carboxymethylcellulose (BDH Ltd) were prepared daily by adding the dry powder to vigorously stirred buffer (Ultra-Turrax, TP18/2N). Both were prepared at the highest concentration required, diluted as necessary, and maintained at $37^{\circ} \mathrm{C}$ in gently stirred, closed flasks for at least two hours before use, to ensure the attainment of stable viscosity. All incubation media contained glucose $(28 \mathrm{mmol})$ unless otherwise stated, and were gassed continuously with $95 \% \mathrm{O}_{2}$ : $5 \% \mathrm{CO}_{2}$.

The viscosities of the guar and sodium carboxymethylcellulose (CMC) dispersions were determined on a rotary viscometer (Contraves Rheomat 15) at a continuous shear rate of $50 / \mathrm{s}$ wherever possible.

\section{MEASUREMENT OF MUCOSAL DIFFUSION}

\section{BAR R IER}

The resistance to diffusion of the mucosal barrier was measured using a modification of the technique developed by Diamond ${ }^{9}$ for the measurement of unstirred layers in gall bladder, and later applied to intestine by other workers. ${ }^{10} 11$ The method depends on the accurate measurement of the half-time for the establishment of an osmotic induced potential, which results from the application of an osmotic load to the mucosal surface. ${ }^{12}$ When a suitable intestinal preparation is suddenly transferred to a medium containing a solute at an appropriate concentration, the osmotic induced potential is established only as rapidly as the solute can diffuse up to the mucosal surface. The effective thickness of the unstirred layer across which the solute must diffuse can be calculated using the following equation. ${ }^{13} \mathrm{~g}$

$$
d=\left(\frac{D^{.} t_{t}}{0.38}\right)^{t}
$$

where $d=$ unstirred layer thickness (microns), $t \frac{1}{2}=$ half-time for the establishment of new potential (seconds), $\mathrm{D}=$ diffusion constant for solute $\left(\mathrm{cm}^{-2} / \mathrm{s}\right)$. In this study, cannulated sacs were rapidly transferred from a tube containing the normal incubation medium to one containing medium plus $50 \mathrm{mmol}$ mannitol. The transmural potential difference was monitored with $\mathrm{KCl}$ /Agar bridges led via calomel half-cells to a Vibron Electrometer (model 33C) with attached chart recorder (Servoscribe IS) runing at $120 \mathrm{~mm} / \mathrm{min}$. All incubation media contained glucose $(28 \mathrm{mmol})$. Under these conditions the osmotic induced potential had been previously shown to be linearly related to mannitol concentration up to at least $150 \mathrm{mmol}$.

\section{OXYGEN CONSUMPTION MEASUREMENTS}

The metabolic activities of tissue samples preincubated with and without guar gum were compared by means of Warburg manometry. Everted sacs from adjacent lengths of jejunum were incubated for 15 minutes in the presence and absence of guar gum. Everted rings of tissue were then prepared from each sac, and their oxygen consumptions were measured in paired Warburg manometers. ${ }^{14} \mathrm{QO}_{2}$ values were subsequently calculated for the dried tissue samples.

\section{EXPERIMENTAL DESIGN}

To study the effect of guar gum or CMC on serosal glucose transport, four sacs were prepared from each animal and allotted at random to flasks containing either gum-free medium, or one of three concentrations of gum, such that the apparent viscosities of the media covered the range $1-110 \mathrm{mPa} \cdot \mathrm{s}(\mathrm{cP})$.

To observe the effect of a single concentration of guar gum on glucose transport over a range of concentrations, 10 sacs were prepared from each animal, adjacent pairs being assigned to test and control flasks by means of a latin square design. Test flasks contained buffer, guar gum $(0.25 \%$, viscosity ca. $16 \mathrm{mPa} \cdot \mathrm{s}(\mathrm{cP})$ ) and glucose at a concentration of $5,10,20,35$, or 50 mmol. Control flasks were identical except that guar gum was omitted from the medium.

After incubation the sacs were rinsed in glucosefree buffer, the serosal solutions were collected in pre-weighed glass vials, and the tissue was dried overnight at $80^{\circ} \mathrm{C}$. Serosal solutions were weighed and assayed for glucose by the GOD/PERID spectrophotometric method (Boehringer Mannheim). The total serosal glucose transport for each sac was calculated and expressed in terms of tissue dry weight.

In experiments designed to study the effect of preincubation in guar containing media, four sacs were prepared and alloted in random pairs to glucose-free media with or without guar gum $(0.25 \%$, viscosity ca. $16 \mathrm{mPa} \cdot \mathrm{s}(\mathrm{cP}))$, and incubated 
Table 1 Serosal glucose transport in presence of guar gum and CMC

\begin{tabular}{lcc}
\hline & $\begin{array}{c}\text { Viscosity } \\
(\mathrm{mPa} \cdot \mathrm{s})\end{array}$ & $\begin{array}{l}\text { Glucose transport } \\
(\mu \mathrm{mol} / \mathrm{g} / 30 \mathrm{~min})\end{array}$ \\
\hline $\begin{array}{l}\text { Guar gum concentration* } \\
(\% \mathrm{w} / \mathrm{v})\end{array}$ & \\
0 & 1 & $31 \pm 10$ \\
$0 \cdot 1$ & 3 & $21 \pm 7$ \\
$0 \cdot 25$ & 16 & $10 \pm 5$ \\
0.5 & 104 & $8 \pm 2$ \\
& & \\
CMC concentrationt $\dagger$ & & \\
$(\% \mathrm{w} / \mathrm{v})$ & 1 & $51 \pm 13$ \\
0 & 2 & $42 \pm 6$ \\
0.1 & 25 & $15 \pm 3$ \\
0.45 & 112 & $9 \pm 3$ \\
0.7 & & \\
\hline
\end{tabular}

-Values are expressed as means and standard error of the mean for seven animals, $P<0.01$ determined by analysis of variance. $1 \mathrm{mPa} \cdot \mathrm{s}=$ $1 \mathrm{cP}$.

tValues are expressed as means and standard errors of the mean for five animals. $\mathrm{P}<0 \cdot 05$ determined by analysis of variance. $1 \mathrm{mPa} \cdot \mathrm{s}=1 \mathrm{cP}$. All mucosal media contained glucose $(28 \mathrm{mmol})$.

for 15 minutes. The four sacs were then rinsed, and incubated for another 15 minutes in guar-free medium containing glucose $(28 \mathrm{mmol})$, so that they were all exposed to an identical mucosal medium during glucose absorption. An average value for glucose transport was then calculated for each pair of sacs.

For unstirred layer measurements, three cannulated sacs were prepared and randomly assigned as follows. For 'sac 1' (Table 2) the measurement was made as soon as a stable transmural potential difference was established in gum-free medium.

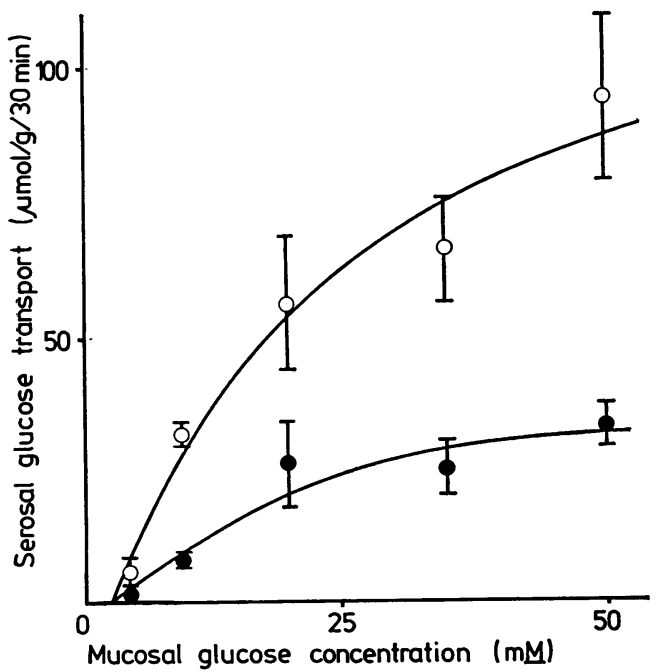

Fig. 1 Comparison of serosal glucose transport in paired sacs (five animals) in the presence (O) and absence (O) of guar gum $(0.25 \%)$ at various concentrations of glucose. Curves fitted by eye.
'Sac 2' was incubated for 15 minutes in gum-free medium before measurement, and 'sac 3' was preincubated for 15 minutes in a medium containing either guar gum $(0.5 \% \mathrm{w} / \mathrm{v}$, viscosity ca. $100 \mathrm{mPa} \cdot \mathrm{s}$ (cP)), or in CMC $(0.7 \% \mathrm{w} / \mathrm{v}$, viscosity ca. $110 \mathrm{mPa} \cdot \mathrm{s}$ (cP)).

\section{Results}

\section{GLUCOSE TRANSPORT IN PRESENCE OF GUAR GUM AND SODIUM CARBOXYMETHYL- \\ CELLULOSE}

The presence of either guar gum or CMC in the mucosal medium was associated with a significant reduction in serosal glucose transport (Table 1). For both types of gum, there was a steep decline in glucose transport as the apparent viscosity of the mucosal media rose from 1 to $20 \mathrm{mPa} \cdot \mathrm{s}$ (cP), with a levelling off thereafter. Figure 1 illustrates the inhibition of glucose transport by guar gum $(0 \cdot 25 \%)$ over a range of mucosal glucose concentrations. Note that the use of a relatively long incubation time, coupled with the evident metabolism of glucose by the sacs, renders a rigorous kinetic analysis of these data inapplicable.

Using the two-stage incubation procedure, it was demonstrated that the inhibitory effects of guar gum

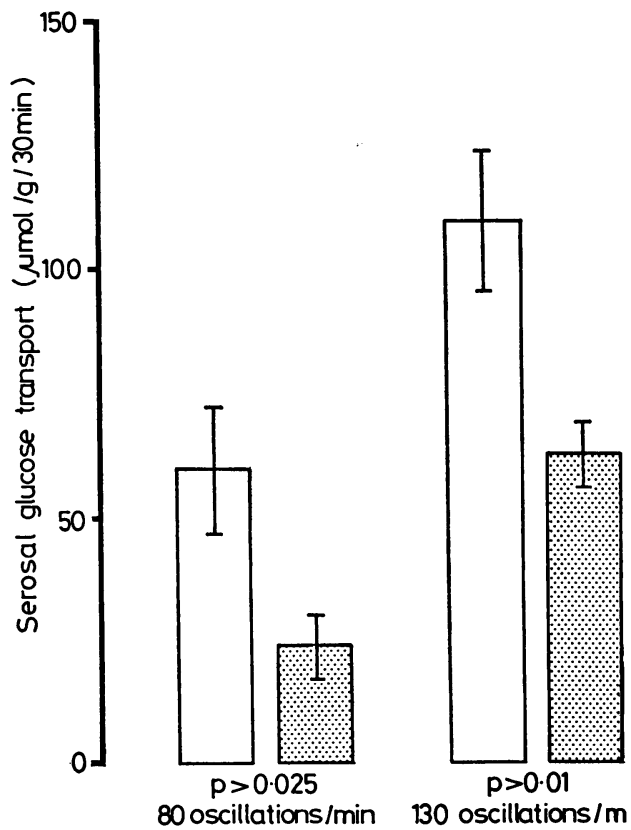

Fig. 2 The effect of increased mucosal stirring (incubator shaking rate) on serosal glucose transport in paired sacs (six animals) with (stippled box) and without mucosal guar gum $(0 \cdot 25 \%)$. 
Table 2 Apparent thickness of mucosal unstirred layer in freshly isolated jejunal sacs compared with sacs preincubated with guar gum or CMC

\begin{tabular}{|c|c|c|c|c|c|}
\hline \multirow[b]{3}{*}{$\begin{array}{l}\text { a. Effect of guar gum ( } 13 \text { animals) } \\
\text { b. Effect of CMC (five animals) }\end{array}$} & \multicolumn{5}{|c|}{ Unstirred layer thickness $(\mu)$} \\
\hline & Sac 1* & & Sac $2 \dagger$ & & Sac 3 \\
\hline & $\begin{array}{l}359 \pm 21 \\
377 \pm 22\end{array}$ & $\begin{array}{l}\mathrm{P}<0.01 \\
\mathrm{NS}\end{array}$ & $\begin{array}{l}317 \pm 15 \\
346 \pm 12\end{array}$ & $\begin{array}{l}\mathbf{P}<0.005 \\
\mathbf{P}<0.005\end{array}$ & $\begin{array}{l}468 \pm 25 \\
402 \pm 12\end{array}$ \\
\hline
\end{tabular}

were persistent, in that sacs exposed to the material in a preliminary incubation showed a significant reduction in transport from $22 \pm 4 \mu \mathrm{mol} / \mathrm{g} / 30 \mathrm{~min}$ (control sacs) to $9 \pm 2 \mu \mathrm{mol} / \mathrm{g} / 30 \mathrm{~min}$ (test sacs), $\mathbf{P}<0.025$.

It was also shown that, even when inhibited by guar gum in the mucosal medium, glucose transport could be stimulated by increasing the stirring rate during incubation. Figure 2 illustrates the behaviour of test and control sacs incubated with and without guar gum $(0.25 \%)$ at two different shaking rates. Increasing the shaking rate from 80 to 130 oscillations per minute roughly doubled the glucose transport in both cases.

\section{MEASUREMENT OF MUCOSAL DIFFUSION}

BARRIER AND INFLUENCE OF GUAR GUM AND SODIUM CARBOXYMETHYLCELLULOSE

Consistent changes were observed in the apparent thickness of the mucosal unstirred layer in sacs incubated with or without guar gum before measurement (Table 2a). Compared with freshly prepared sacs, those incubated for 15 minutes in guar-free Krebs showed a significant reduction of about $10 \%$. However, test sacs incubated for 15 minutes with guar gum possessed a mucosal unstirred layer nearly $50 \%$ thicker than control sacs incubated in guar free buffer. A similar trend was observed in sacs incubated with CMC (Table 2b).

\section{OXYGEN CONSUMPTION IN EVERTED} INTESTINE PREINCUBATED WITH GUAR GUM The $\mathrm{QO}_{2}$ values for paired samples of tissue, incubated for 15 minutes with or without guar gum $(0.25 \%)$ were measured for six animals. In all cases the oxygen uptake was linear for at least 30 minutes, and the $\mathrm{QO}_{2}$ for tissue preincubated with guar gum $(8.1 \mu \mathrm{mol} / \mathrm{min} / \mathrm{g})$ was not significantly different from tissue preincubated in guar-free medium $(7.5 \mu \mathrm{mol} /$ $\min / g$ ).

\section{Discussion}

In this study we have shown that the intestinal transport of glucose in vitro is substantially reduced in the presence of low concentrations of guar gum, the main constituent of which is guaran, a naturally occurring polymer of D-mannopyranose units, bearing single membered $\mathrm{D}$-galactopyranose branches. A direct interaction between the very large polysaccharide chains and glucose transport sites in the absorptive cell membrane is improbable, and, although the presence of physiologically active impurities in the guar gum cannot be ruled out, this would not account for the similar inhibitory effect of sodium carboxymethylcellulose, a synthetic gum with a different chemical structure. We believe therefore that a specific biochemical inhibition of glucose transport is an unlikely explanation of the present results; nor was any evidence obtained for an impairment of aerobic metabolism brought about by the unusual incubation conditions.

The restoration of glucose transport in the presence of guar gum, to control levels, brought about by increased stirring during incubation (Fig. 2), seems inconsistent with any reduction in the glucose transport capacity of the tissue itself, but this result can be readily explained in terms of a reduction in the effectiveness of the mucosal diffusion barrier, brought about by a thinning of the unstirred water layer overlying the intestinal villi. The importance of the intestinal unstirred layer as a modifier of transport kinetics has been stressed recently by several workers. ${ }^{15} 1617$

Taking the figures for guar gum (Table 2a), a value of $359 \pm 21 \mu$ was obtained for the unstirred layer thickness of freshly prepared sacs measured under unstirred conditions. This figure is substantially higher than that obtained for rat jejunum by previous workers ${ }^{10}(198 \pm 5 \mu)$ but it is comparable with that of about $300 \mu$ measured in rabbit intestine, ${ }^{11}$ and substantially less than the figure of $632 \pm 24 \mu$ measured in vivo in human subjects. ${ }^{18}$ Such discrepancies presumably reflect morphological differences in the various preparations as well as differences of experimental technique.

Incubation for 15 minutes in guar-free buffer led to a significant reduction of about $10 \%$ in the thickness of the unstirred layer. This finding is 
consistent with the report of Westergaard and Dietschy, ${ }^{11}$ who observed a similar reduction in the unstirred layer of rabbit intestine after incubation in vitro, and who showed this to be accompanied by swelling of the villi with a consequent obliteration of the intervillous spaces due to fluid accumulation. In the present studies, incubation of the intestine for 15 minutes in a medium containing guar gum led to an increase in the apparent thickness of the unstirred layer of about $48 \%$ when compared with sacs preincubated without guar, or $30 \%$ compared with the freshly prepared sacs. Similar, though less pronounced, changes were observed in the apparent unstirred layer thickness of sacs incubated with CMC (Table 2b).

We propose that the inhibition of glucose transport observed in the present study is best explained by an increase in the resistance of the mucosal diffusion barrier brought about by the greater viscosity of incubation media containing polysaccharide gums. It is important to realise that the unstirred layer measurements quoted here and in other publications are not to be taken too literally. In reality, there is no stationary water layer sharply divided from the bulk phase, rather there is a region of progressively diminished stirring, tending toward immobility at the mucosal surface. The calculated thickness corresponds to an idealised immobile film of water having an equivalent resistance. The presence of long polymer chains in the incubation medium confers upon it some of the properties of a gel, with a corresponding decline in the mobility of the fluid layers surrounding and overlying the intestinal villi.

A second possible factor limiting the diffusion of solutes in media containing viscous gums, is the obstruction caused by the molecules themselves. In a gel, the molecular chains form a complex threedimensional lattice extending throughout the aqueous phase. Assuming that there is no interaction between the solute and the polymer, and that the diffusing molecule is small in relation to the intersegmental distance of the lattice, then its mobility is restricted as a result of collisions with the polymer which increase the effective diffusion path length. This restricted diffusion may be expressed by the formula of Mackie and Meares: ${ }^{19}$

$$
\frac{D_{p}}{D_{o}}=\left[\frac{(1-v)}{(1+v)}\right]^{2}
$$

where $D_{p}$ and $D_{o}$ are the diffusion coefficients of the molecule in the polymer and water respectively, and $\mathrm{v}$ is the volume fraction of the polymer in solution.

For a gel having a polymer volume fraction of $1 \%$, the diffusion coefficient will be reduced to about $96 \%$ of its value in water. In calculating the apparent unstirred layer thickness we have ignored this effect and assumed a constant value for the diffusion coefficient of mannitol, regardless of the presence of guar or CMC. This is partly because of the low concentration of gum used, and partly because under the conditions of measurement, the concentration of gum at the mucosal surface is not known with accuracy. Nevertheless, the restrictive effect of the gel structure must make some contribution to the increased resistance of the mucosal diffusion barrier, and this may have to be taken into account when considering the effects of large doses of gum in vivo.

The possible importance of viscous gums in modifying intestinal absorption of nutrients, as opposed to their effects on gastric motility, ${ }^{4}$ will need to be assessed in additional studies. The present work shows that, in appropriate circumstances, the presence of gum in contact with the intestinal mucosa exerts a considerable influence upon transport. In the intact animal there is, presumably, an interaction of the two effects, with their relative importance dependent on composition of the diet. At present little is known about stirring rates in the intestine, or about the viscosity of the normal intestinal contents in man and the way this varies in response to changes in dietary composition. Nor do we know the possible importance of other factors besides viscosity in determining the behaviour of nonabsorbable materials in the gut. In this study we have shown that the guar gum need not be in the bulk phase to exert its effect, but need only be present in a superficial film after preincubation of the intestine in the gum. This may indicate some degree of interaction between the guar and mucopolysaccharides of the mucosal surface, which perhaps help 'anchor' the surface layer for a period. If this is so, the chemical structure of a particular gel-forming gum may help determine its effectiveness in modifying the intestinal unstirred layer. It is hoped that these approaches may prove effective in determining the importance of the dietary fibre in the normal human diet, as well as helping contribute to the development of an optimal diet for the management of diabetes in man.

\section{References}

'Jenkins D. Dietary fibre, diabetes and hyperlipidaemia: progress and prospects. Lancet 1979; 2: 1287-9.

${ }^{2}$ Jenkins D, Goff D, Leeds A, et al. Unabsorbable carbohydrates and diabetes: decreased post-prandial hyperglycaemia. Lancet 1976; 2: 172-4.

${ }^{3}$ Jenkins D, Wolever T, Leeds A, et al. Dietary fibres, fibre analogues, and glucose tolerance: importance of viscosity. $\mathrm{Br}$ Med J 1978; 1 : $1392-4$.

${ }^{4}$ Holt S, Heading R, Carter D, Prescott L, Tothill P. Effect of gel fibre on gastric emptying and absorption of glucose and paracetamol. Lancet 1979; 1: 636-9. 
${ }^{5}$ Taylor R. Gastric emptying, fibre, and absorption. Lancet 1979; 1 : 872.

${ }^{6}$ Leeds A. Gastric emptying, fibre, and absorption. Lancet 1979; 1: 872-3.

${ }^{\top}$ Kawatra B, Garcha J, Wagle S. Effect of feeding guar gum and guar meal on nitrogen balance and on the intestinal absorption of amino acid. J Res Punjab Agric Univ 1969; 8: 227-31.

${ }^{8}$ Southgate D. Fibre and the other unavailable carbohydrates and their effects on the energy value of the diet. Proc Nutr Soc 1973; 32: 131-6.

${ }^{\circ}$ Diamond J. A rapid method for determining voltageconcentration relations across membranes. $J$ Physiol 1966; 183: 83-100.

${ }^{10}$ Wilson F. Dietschy J. The intestinal unstirred layer: its surface area and effect on active transport kinetics. Biochem Biophys Acta 1974; 363: 112-26.

${ }^{11}$ Westergaard H, Dietschy J. Delineation of the dimensions and permeability characteristics of the two major diffusion barriers to passive mucosal uptake in the rabbit intestine. J Clin Invest 1974; 54: 718-32.
${ }^{12}$ Smyth D, Wright E. Streaming potentials in the rat small intestine. J Physiol 1966; 182: 591-602.

${ }^{13}$ Dainty J, House C. 'Unstirred layers' in frog skin. $J$ Physiol 1966; 182: 66-78.

${ }^{14}$ Umbriet W, Burris R, Stauffer J. Manometric techniques and tissue metabolism 1951; 2nd edn. Burgess, Minneapolis.

${ }^{15}$ Dietschy J, Sallee V, Wilson F. Unstirred water layers and absorption across the intestinal mucosa. Gastroenterology 1971; 61: 932-4.

${ }^{16}$ Winne $D$. The influence of unstirred layers on intestinal absorption. Excerpta Med Int Congr Ser 1977; 391: $58-64$.

${ }^{17}$ Dainty J. Water relations of plant cells. Adv Bot Res 1963; 1: 279-326.

${ }^{18}$ Read N, Barber D, Levin R, Holdsworth C. Unstirred layer and kinetics of electrogenic glucose absorption in the human jejunum in situ. Gut $1977 ; 18$ : 865-76.

${ }^{19}$ Mackie J, Meares P. The diffusion of electrolytes in a cation-exchange resin membrane. I. Theorectical. Proc $R$ Soc 1955; 232 A: 498-509. 